\title{
Efficient 3D-3D Vascular Registration Based on Multiple Orthogonal 2D Projections
}

\author{
Ho-Ming Chan and Albert C. S. Chung \\ Dept. of Computer Science, Hong Kong University of Science and Technology, HK \\ \{hmchan, achung\}@cs.ust.hk
}

\begin{abstract}
In this paper, we present a new and efficient multi-modal 3D-3D vascular registration algorithm, which transforms the 3D-3D registration problem into a multiple 2D-3D vascular registration problem. Along each orthogonal axis, projected 2D image from a segmented binary 3D floating volume is compared with maximum intensity projection (MIP) image of the reference volume. At the preprocessing stage of the floating image volume, vessels are segmented and represented by a number of spheres with centers located at the skeleton points of the vessels and radii equal to the distance from the skeleton points to their closest boundary. To generate projected images from the binary $3 \mathrm{D}$ volume, instead of using the conventional ray-casting technique, the spheres are projected to the three orthogonal projection planes. The discrepancy between the projected image and the reference MIP image is measured by a relatively simple similarity measure, sum of squared differences (SSD). By visual comparison, we found that the performances of our method and the Mutual Information (MI)-based method are visually comparable. Moreover, based on the experimental results, our method for 3D-3D vascular registration is more computationally efficient than the MI-based method.
\end{abstract}

\section{Introduction}

Multi-modal image registration is a key for the integration of the complementary image information available in different modalities by aligning the images. Images acquired by different medical imaging modalities provide useful complementary information. For example, time-of-flight magnetic resonance angiographic (TOFMRA) or 3D rotational angiographic (RA) images provide anatomical information; phase contrast (PC) MRA images provide flow information in the vessels. As such, matching between TOF- and PC-MRA or RA and PC-MRA images can provide flow and structural information in an integrated 3D volume, which is very useful for the physicians.

To correctly align two images, we need a similarity measure to determine how well an image matches against another image through a hypothesized spatial transformation. Most of the similarity measures can be classified into two categories: feature-based or intensity-based. In Section 1.1, we will briefly introduce these similarity measures, and discuss their advantages and disadvantages. In general, the intensitybased method is more accurate. On the other hand, the feature-based method is more computationally efficient [16]. In this paper, we extend the early version of our work 
in [3] from 2D-3D to 3D-3D registration, and will also compare our proposed method with the Mutual Information (MI)-based registration method [6], [7], which is one of the most commonly used intensity-based methods in the medical image analysis field.

\subsection{Previous Works}

Over the past few years, many intensity-based similarity measures have been introduced, for example sum of squared differences (SSD), correlation coefficients (CC), correlation ratio (CR) [15] etc. The most popular and widely used one is the information-theoretic similarity measure, namely MI [6], [7]. It does not require segmentation and only makes use of the assumption of statistical dependence between the two images, and has been successfully applied to many multi-modality combinations.

The feature-based registration algorithms extract features such as surfaces, curves [13], [24], [25] or skeletons [11], [12], [14] at the preprocessing steps. They are faster than intensity-based methods but comparatively less accurate [16]. Also, the extraction of skeletons, for example, can be sensitive to noise [17]. Therefore, registration algorithms using skeletons as the only feature for registration can be sensitive to noise as well. On the other hand, the surface or curve extraction and representation can be complicated and would also affect the registration accuracy.

Recently, Chung and et al. [1], [2] used a pair of precisely registered images to build the expected joint intensity or class histogram and employed KLD [4], [5] to measure the discrepancy between observed and expected joint intensity or class histograms. It makes use of the a priori knowledge of the expected joint intensity or class histogram to guide the transformation towards the expected outcome.

\section{Our 3D-3D Vascular Registration Algorithm}

We will provide some background information in the following subsections before we introduce our registration algorithm. For the conventional MI-based registration method, a joint intensity histogram is built at each iteration during the registration process. Within the overlapping region of the floating and reference images, each voxel at the floating image is transformed to the coordinate frame of the reference image and the corresponding intensity bin of the histogram is incremented. Generation of a histogram using the intensity pairs in the overlapping region can be very time-consuming, particularly when the size of image volume is large. A large portion of the registration computational time can be spent on the building of joint intensity histograms. Therefore, we propose to transform the 3D-3D registration problem into a multiple $2 \mathrm{D}-3 \mathrm{D}$ registration problem and compare the projection images perpendicular to the three orthogonal axes until the registration process terminates. We first generate three MIP images of the reference volume along each of the axes. At each iteration, we generate three projected images from the segmented binary floating volume to compare with the corresponding MIPs generated before. An algorithm for generating binary projected images is described in Section 2.2. In order to generate projected images from a binary volume efficiently, some preprocessing of the floating volume is needed. 


\subsection{Preprocessing of Floating Volume}

Before the registration process, an isotropic volume is built so that the new voxel size is equal to the original in-plane voxel size, and the intensities of voxels between slices are estimated by using the trilinear interpolation. Vessels are then segmented by using the global thresholding method and skeletons are extracted [18] with the modified weighted metric $<1, \sqrt{2}, \sqrt{3}>$. (Some advanced segmentation method can be used. But, it is not the focus of this paper.) The skeleton points (within a $7 \times 7 \times 7$ window) are connected using the depth first search. The main vessel that we are interested is usually the largest connected component containing most skeleton points. By using the extracted skeleton, the original binary volume can be represented and reconstructed by the set of spheres with the coordinates of skeleton points as centers and their distance transform values (their distances to the closest boundary) as radii [22]. Note that a rectangular region of interest containing the main vessels is selected in order to shorten the computational time.

\subsection{Generation of Projected Image from a Binary Volume}

In order to mimic the MIP images, the whole projected image is randomly initialized to one of the background intensities of the MIP image. The background intensities of the MIP image can be easily obtained from the boundary pixels.

Given the rigid transformation matrix, the center of each sphere of the main vessel is projected to each of the three projection planes under orthogonal projective geometry. The intensities of all pixels with its distance to the projected center less than the corresponding radius are assigned to 255 .

A pair of the projected images generated (a) by projection of spheres and (b) by using the ray-casting technique is shown in Fig. 1. The differences of the resulting images are negligible. Our method not only produces similar image compared to the ray-casting technique, but also it is more efficient. A comparison on computational efficiency between the two methods will be presented in Section 3.1.

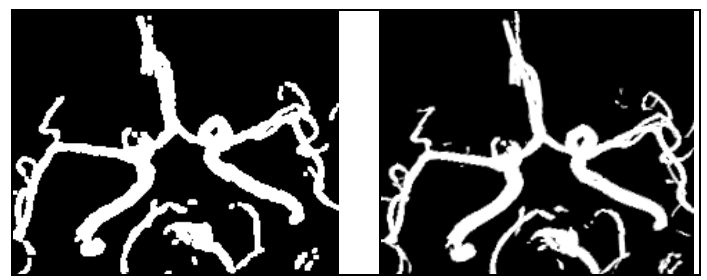

Fig. 1. Projected images from a binary volume generated by (a) projection of spheres (left) and (b) the ray-casting technique (right).

\subsection{Sum of Squared Differences as Similarity Measure}

Vessels in both MIP images and projected binary images have high intensity values. Sum of squared differences (SSD), which is a simple similarity measure, can be employed to measure the difference between a 2D MIP image, denoted by $U$, and the 
corresponding binary projected image from the segmented floating volume, denoted by $V$. The SSD between the two images is given by

$$
\operatorname{SSD}(U, V)=\sum_{i \in \Phi}(U(i)-V(i))^{2}
$$

where $\Phi$ represents the image domain. When the two images $U$ and $V$ are precisely aligned, the value of SSD is expected to be minimum. We use the sum of the three SSDs for each axis as the similarity measure to guide our registration process.

It is interesting to point out that, from our experience, the similarity measures: MI and KLD also work well in this problem. However, they require extra computations such as building joint intensity or class histograms, normalizing the histograms and calculating marginal distributions (for MI). Moreover, the bin sizes of the joint intensity histograms may affect the registration result.

\subsection{Outline of Registration Process}

During the registration process, the floating image is the 3D PC-MRA volume while the reference image is the 3D TOF-MRA volume, which is fixed in the 3D space. At each iteration, three binary projected 2D images, along each orthogonal axes, of the segmented PC-MRA volume for the current pose is generated by the method mentioned in Section 2.2. The goal is to find the optimal rigid transformation $\hat{T}$ by minimizing the value of sum of SSDs (see Equation (1)) between the binary projected images and their corresponding MIP images,

$$
\hat{T}=\arg \min _{T}\left(S S D\left(U_{x y}, V_{x y}(T)\right)+S S D\left(U_{x z}, V_{x z}(T)\right)+S S D\left(U_{y z}, V_{y z}(T)\right)\right) .
$$

Powell's method [10] is used to iteratively search for the minimum value of the sum of SSDs along each parameter (in 3D, the rigid transformation has three translational and three rotational parameters) using Brent's method [10]. The algorithm halts when the percentage change of the sum of SSDs is below a user-specified threshold. We set this threshold to $0.001 \%$ in our program. The flow chart of our registration algorithm is shown in Fig. 2.

\section{Results and Comparisons}

In this section, we show the results of the 3D-3D rigid vascular registration algorithm presented in Section 2 and also compare its accuracy and computational efficiency with the MI-based method. We used three pairs of TOF- and PC-MRA datasets for this experiment to study the performance of the SSD-based method and selected one of the three pairs (Case 1) for comparison with the MI-based method. The specification of the MRA dataset is listed in Table 1. 


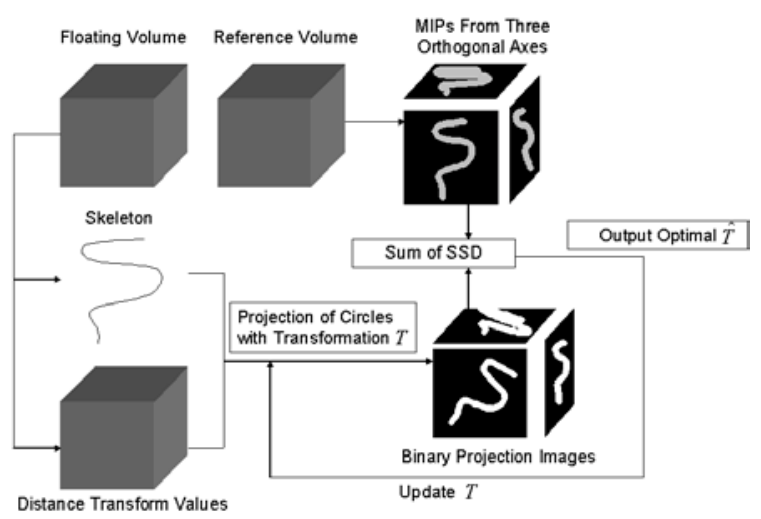

Fig. 2. A flow chart of our registration algorithm

Table 1. Specifications of the datasets used for evaluation of our registration algorithm.

\begin{tabular}{|c|c|c|c|c|}
\hline \multirow{2}{*}{$\begin{array}{l}\text { Case } \\
\text { No. }\end{array}$} & \multicolumn{2}{|c|}{ Floating Volume } & \multicolumn{2}{|c|}{ Reference Volume } \\
\hline & $\begin{array}{l}\text { Dimensions } \\
(\mathrm{H} \text { x W x D })\end{array}$ & Voxel Size (mm) & $\begin{array}{l}\text { Dimensions } \\
(\mathrm{H} \text { x W x D })\end{array}$ & Voxel Size (mm) \\
\hline 1 & $512 \times 512 \times 64$ & $0.390625 ; 0.390625 ; 1$ & $512 \times 512 \times 80$ & $0.390625 ; 0.390625 ; 0.75$ \\
\hline 2 & $512 \times 512 \times 32$ & $0.390625 ; 0.390625 ; 0.9$ & $512 \times 512 \times 60$ & $0.410156 ; 0.410156 ; 0.95$ \\
\hline 3 & $512 \times 512 \times 64$ & $0.390625 ; 0.390625 ; 1$ & $512 \times 512 \times 60$ & $0.390625 ; 0.390625 ; 0.95$ \\
\hline
\end{tabular}

\subsection{Probing Experiments}

The first pair of the volumes was used in this experiment. The resulting transformation obtained by using each method was assumed to be their corresponding optimal transformations. The probing results of the objective functions are shown in Fig. 3 . The performances of SSD and MI are comparable when the floating volume was shifted between $-10 \mathrm{~mm}$ to $10 \mathrm{~mm}$ against $\mathrm{Tx}$, Ty, and was rotated between $-18^{\circ}$ to $18^{\circ}$.

The computational times of all the evaluations in the probing experiments presented in this subsection are summarized as follows. For the SSD-based method, the mean and standard deviation are $0.19 \mathrm{~s}$ and $0.02 \mathrm{~s}$ respectively while for the MI-based method, the mean and standard deviation are $17.79 \mathrm{~s}$ and $1.41 \mathrm{~s}$ respectively.

\subsection{A Study on Computational Time of Our Registration Algorithm}

In order to compare the computational efficiency between our method and the raycasting method for generating a projected image from binary volume, we generated 1000 projected images from a binary volume using both methods. The ray-casting method takes 1.34 seconds on average whereas our method takes 0.07 seconds on average, which is about 20 times faster than the ray-casting method. The computational times of the whole registration process for SSD-based and the MI-based method are listed in Table 2. All experiments done in this paper were run on a 1.7GHz Pentium IV PC with 768M RAM. Based on the performance of probing experiments and the first dataset, it shows that our method can run faster than the MI- 
based method by about 100 times. Note that the MI-based method failed to converge in cases 2 and 3 .

Table 2. Comparisons of the computational time (in sec) for the two registration algorithms.

\begin{tabular}{|l|l|l|l|}
\hline Methods & Case 1 & Case 2 & Case 3 \\
\hline SSD & 36.963 & 43.192 & 26.918 \\
\hline MI & 4705.746 & \multicolumn{2}{|l}{} \\
\cline { 1 - 2 } & \multicolumn{2}{|l}{}
\end{tabular}

\subsection{A Study On Performance of Our Registration Algorithm}

The results are shown in Fig. 4, which shows the MIPs of the registered MRA volumes using the proposed method. Full MIPs are shown here for the visualization purpose only. During the registration, only binary projected images were generated and used. Segmented vascular regions of the binary projected image (at final alignment using the proposed method) are overlaid on the corresponding DSA images (h) and the results are promising.

\section{Discussions and Conclusions}

For the preprocessing of 3D volume, the centerline extraction method proposed by Aylward and et al. [26] does not need any segmentation but initial seek points and scales of each vascular branch are required. In our algorithm, instead of using the thinning algorithm [20] or the centerline extraction algorithm [26], skeletons are extracted by using the distance transforms [18], [22]. With the skeleton points and the distance transform values, the original volume can be recovered exactly [22], which may not be feasible by thinning algorithms or centerline extraction algorithms. Skeletons or centerline extraction can be sensitive to noise [17]. Noisy data may give a lot of undesired skeleton points which can affect the accuracy of the method proposed by Aylward and et al. [26] because they use skeletons only. Our method requires skeletonization as well, but it does not use skeletons as the only feature. Instead of using skeletons only, our method also makes use of the distance transform values for generating binary projected images. Thus, the accuracy of our registration algorithm can be less sensitive to noise.

In our projection-based registration method, instead of projecting all vessel voxels, we draw filled circles with centers located at the projected skeleton points, as mentioned in Section 2.2. The reason is that projecting only vessel voxels may result in some blank horizontal or vertical lines in the projected image, which are undesirable and can be avoided by using our method.

To conclude, we have proposed a new and efficient multi-modal 3D-3D vascular registration method based on the efficient generation of projected images from a binary $3 \mathrm{D}$ volume. The discrepancy between the projected images and the reference MIP images is measured by the sum of squared differences (SSD) similarity measure. Experimental results show that our method is more computationally efficient than the MI-based method while the accuracies of the both methods are comparable. 
Efficient 3D-3D Vascular Registration

307

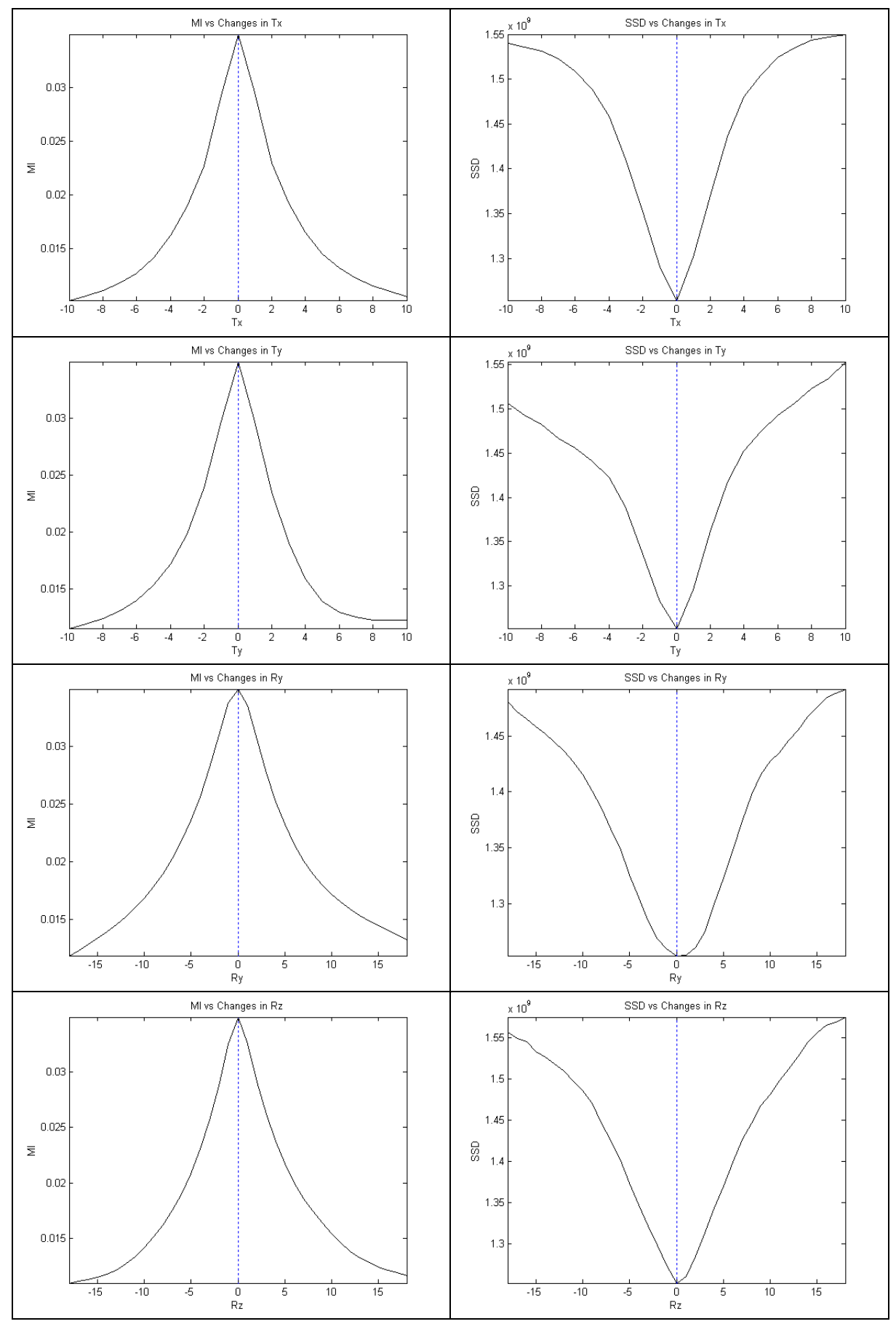

Fig. 3. Probing results of MI(left) and SSD(right) values, in which Tx, Ty, Ry, Rz were varied. 


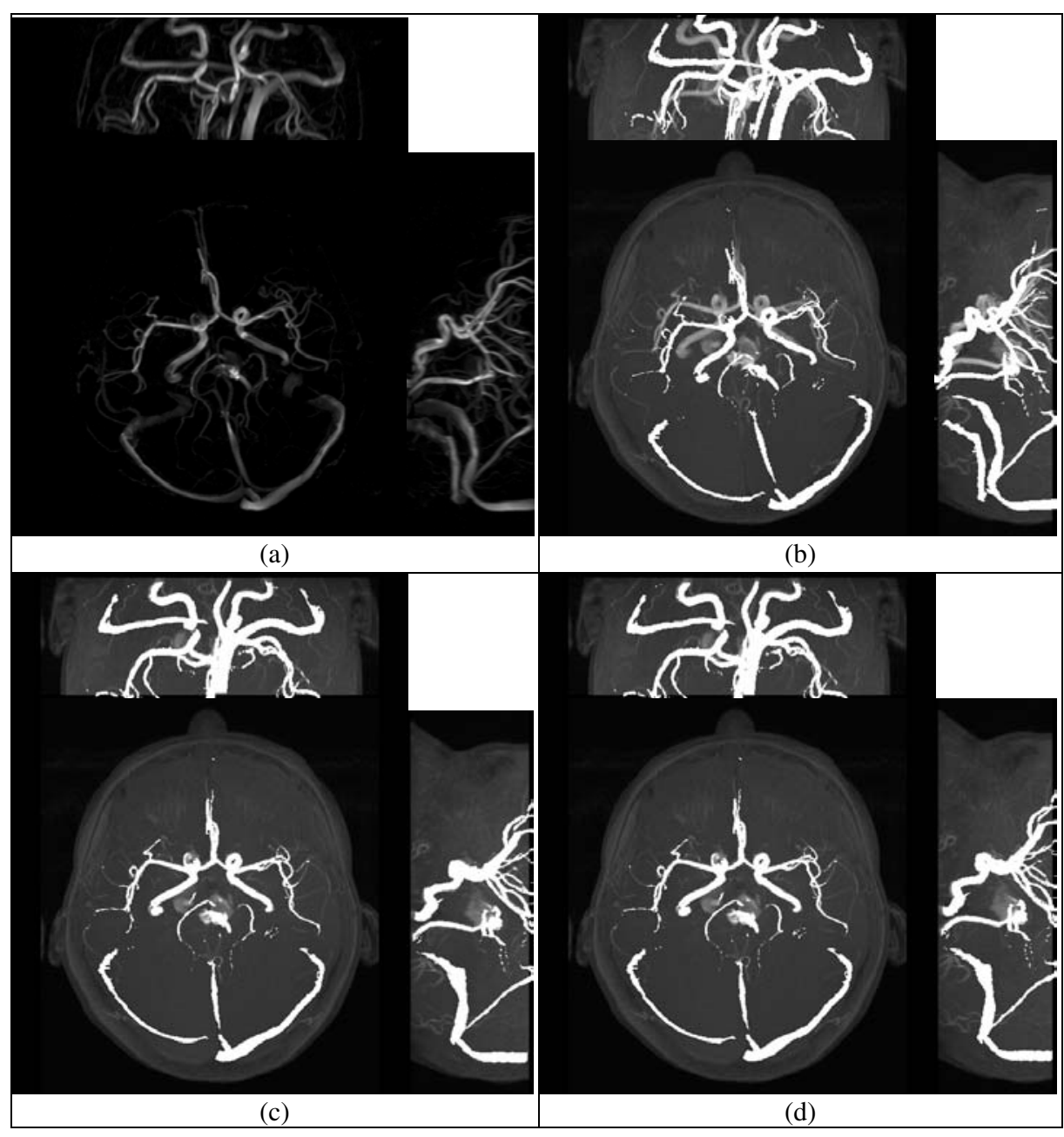

Fig. 4. Results of case 1. (a) MIPs of PC-MRA volume at the initial alignment. (b) Initial image alignment, and binary projected images of PC-MRA volume are overlaid to the TOF-MRA MIP images. (c) Final image alignment by using the SSD-based method, and binary projected images of PC-MRA volume are overlaid to the TOF-MRA MIP images. (d) Final image alignment by using the MI-based method, and binary projected images of PC-MRA volume are overlaid on the TOF-MRA MIP images. 

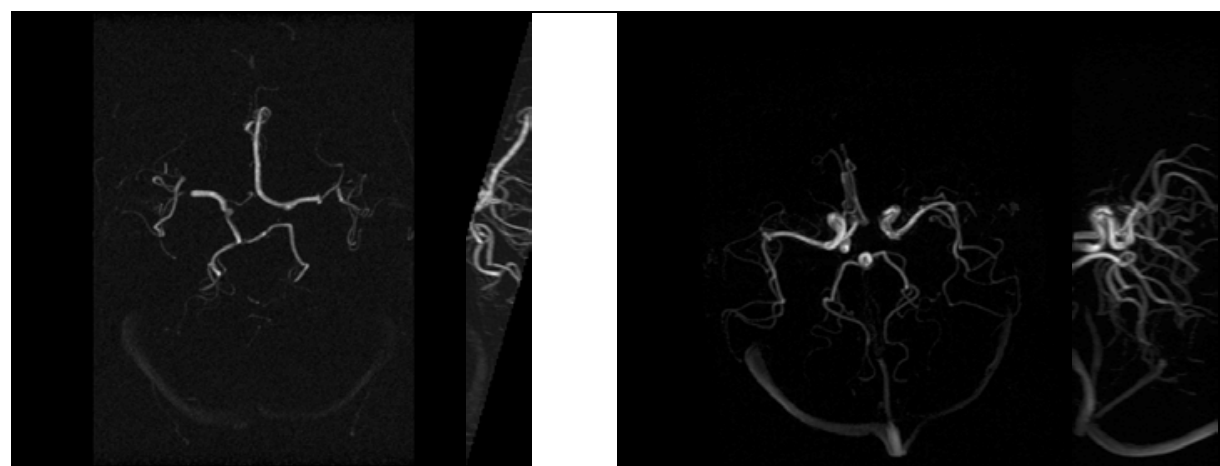

(a)

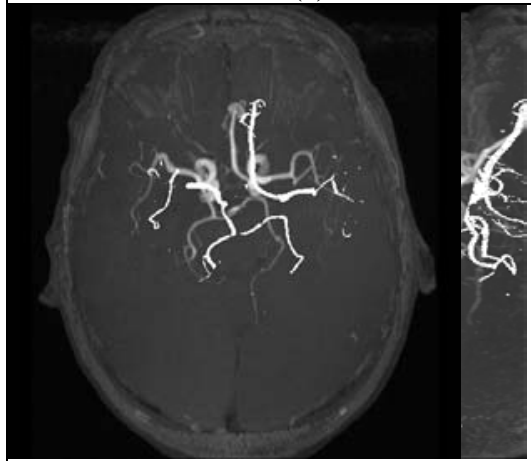

(b)

(c)

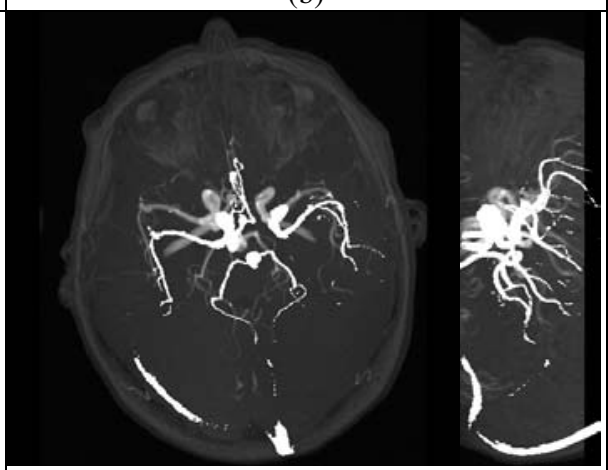

(d)

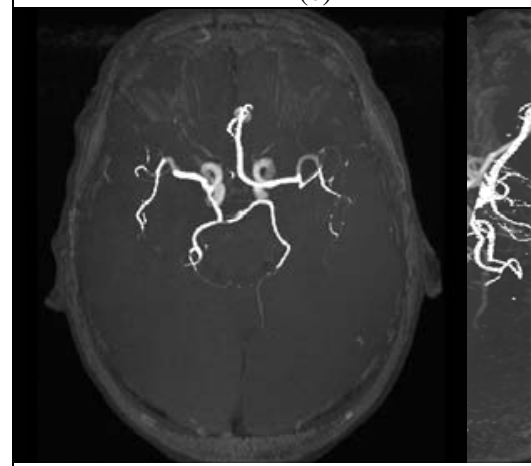

(e)

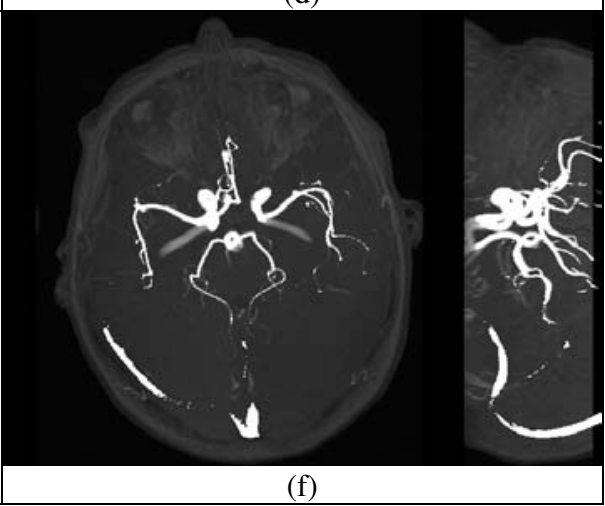

Fig. 5. Results of cases 2 and 3. (a, b) MIPs of PC-MRA volume at initial alignment respectively. (c, d) Initial image alignment, and binary projected images of PC-MRA volume are overlaid to the TOF-MRA MIP images respectively. (e, f) Final image alignment by using the SSD-based method, and binary projected images of PC-MRA volume are overlaid on the TOFMRA MIP images respectively.

\section{References}

1. Albert C.S. Chung, William M. Wells III, and et al., "Multi-modal Image Registration by Minimising Kullback-Leibler Distance", MICCAI, 2002, p. 525-532. 
2. Ho-Ming Chan, Albert C.S. Chung and et al., "Multi-modal image registration by minimizing Kullback-Leibler distance between expected and observed joint class histograms", To appear in CVPR'03.

3. Ho-Ming Chan, Albert C.S. Chung and Simon C.H. Yu, "2D-3D Vascular Registration Algorithm between Digital Subtraction Angiographic (DSA) and Magnetic Resonance Angiographic (MRA) Images", Submitted to MICCAI, 2003.

4. T.M. Cover and J.A. Thomas. Elements of Information Theory. John Wiley \& Sons, Inc., '91.

5. S. Kullback. Information Theory and Statistics. Dover Publications, Inc., 1968.

6. W. Wells, P. Viola, and et al., "Multi-modal Volume Registration by Maximization of Mutual Information", Medical Image Analysis, 1996; 1:32-52.

7. A. Colignon et al., "Automated multi-modality image registration based on information theory", IPMI, p.263-274; Kluwer Academic Publisher, 1995.

8. P. Wyatt and J. Noble, "MAP MRF Joint Segmentation and Registration", MICCAI'02, p.580-587.

9. E. Cosman, "Rigid Registration of MR and Biplanar Fluoroscopy", Master Thesis, Dept. of Electrical Engineering and Computer Engineering, MIT, 2000.

10. W.H. Press, S.A. Teukolsky, and et al., Numerical Recipes in C, 2nd Edition, Cambridge University Press, p.402-405,412-420; Kluwer Academic Publisher, 1995.

11. Y. Kita, D.L. Wilson and et al., "Real-time Registration of 3D Cerebral Vessels to X-ray Angiograms", MICCAI, 1997, p.1125-1133.

12. A. Liu, E. Bullitt and et al., "3D/2D Registration via skeletal near projective invariance in tubular objectives", MICCAI, 1998, p.952-963.

13. J. Feldmar, N. Ayache and et al., "3D-2D projective registration of free-form curves and surfaces", INRIA, Report \# 2434, 1994.

14. J. Feldmar, G. Malandain and et al., "Matching 3D MR Angiography Data and 2D X-ray Angiograms", CVRMed-MRCAS'97, Grenoble, France, p.129-138,

15. A. Roche, G. Malandain, and et al., "Multimodal image registration by maximization of the Correlation Ratio", INRIA, Report \# 3378, 1998.

16. R.A. McLaughlin, J.Hipwell, and et al., "A comparison of 2D-3D intensity-based registration and feature-based registration for neurointerventions", MICCAI, 2002, p.517-524.

17. R. Fisher, S. Perkins, and et al., http://www.dai.ed.ac.uk/HIPR2/skeleton.htm, 2000.

18. N. Gagvani, "Skeletons and Volume Thinning in Visualization", MS. Thesis, Dept. of Electrical and Computer Engineering, Rutgers University, New Brunswick, New Jersey, 1997.

19. A. Shahrokni, H. Soltanian-Zadeh and et al., "Fast skeletonization algorithm for 3-D elongated objects", Proceedings of SPIE, 2001; 4322:323-330.

20. K. Palagyi, R. Sorantin and et al., "A Sequential 3D Thinning Algorithm and Its Medical Applications", IPMI, 2001, p. 409-415.

21. Y. Zhou, A. Toga, "Efficient Skeletonization of Volumetric Objects", IEEE Transactions on Visualization and Computer Graphics, 1999, p.196-209.

22. A. K. Jain, Fundamentals of Digital Image Processing, p.381-389, Prentice Hall, 1989.

23. A. Watt, M. Watt, Advanced Animation and Rendering Techniques: Theory and Practice, Addison-Wesley, Reading, Massachusetts, 1992.

24. X. Pennec, "Toward a generic framework for recognition based on uncertain geometric features", Videre: Journal of Computer Vision Research, 1(2):p.58-87, 1998.

25. C. R. Maurer, Jr., R. J. Maciunas, J. M. Fitzpatrick, "'Registration of head CT images to physical space using multiple geometrical features", Proc. SPIE Medical Imaging 98, San Diego, CA, vol. 3338, 72-80 (Feb 1998).

26. Aylward S, Bullitt E, "Initialization, Noise, Singularities, and Scale in Height-Ridge Traversal for Tubular Object Centerline Extraction", IEEE Transactions on Medical Imaging, Feb, 2002, Pages 61-75 